\title{
NUMERICAL ANALYSIS OF CONFORMAL UC-PBG STRUCTURES
}

\author{
H. R. Khaleel ${ }^{*}$, H. M. Al-Rizzo, and D. G. Rucker
}

Department of Systems Engineering, George W. Donaghey College of Engineering and Information Technology, University of Arkansas at Little Rock, Little Rock, AR 72204, USA

\begin{abstract}
In this paper, the performance of conventional Uniplanar Compact Photonic Band Gap (UC-PBG) structures is investigated under different bending extents. The structure under study is operated as an Artificial Magnetic Conductor (AMC) in which performance is mainly characterized by its resonant frequency and bandwidth. Modelling and numerical analysis have been carried out using CST Microwave Studio simulation software which is based on Finite Integration Technique (FIT). Results show that different bending extents affect the AMC's performance which is specified by a shift to higher resonant frequencies and bandwidth degradation when the degree of bending is increased. Furthermore, we point out some important simulation tips to avoid inaccurate and/or invalid results. This type of study is important to evaluate the performance of such structures for conformal applications. To the best of the authors' knowledge, such type of systematic study is being reported for the first time.
\end{abstract}

\section{INTRODUCTION}

Recent years have witnessed a great interest in Photonic BandGap (PBG) structures due to their various applications in the fields of electromagnetics and optics [1-3]. Their compact size, properties of surface wave suppression which significantly enhances antenna performance $[4,5]$, and more importantly their potential to realize Artificial Magnetic Conductors (AMCs) [6,7] have made PBG structures very popular in the microwave and antenna research communities. AMC which does not exist naturally can be artificially

Received 30 July 2011, Accepted 17 August 2011, Scheduled 19 August 2011

* Corresponding author: Haider R. Khaleel (hrkhaleel@ualr.edu). 
engineered to have in-phase reflection properties over a specified frequency range. AMCs are typically realized based on periodic metallic patterns often termed Frequency Selective Surfaces (FSSs) backed by a grounded substrate.

The in-phase reflection feature is highly desired in antenna applications over Perfect Electric Conductors (PECs) which has a reflection phase of $180^{\circ}$ for a normally incident plane wave. Image theory states that a PEC ground plane causes the antenna's current and its image to cancel each other, i.e., shorts the antenna. This is responsible for dropping the real part of the antenna impedance towards zero ohms, while the imaginary impedance approaches infinity. Thus, a significant amount of the electromagnetic energy is trapped between the antenna and the ground plane; hence the antenna can no longer radiate efficiently [8]. This is the opposite scenario if an AMC is placed instead of PEC due to its reflection of electromagnetic wave with zero phase shift.

AMC was first proposed in 1999 by Sievenpiper et al. [9], which was based on square metallic plates connected to a ground plane through vias, also referred to as "mushroom" structures. Later on, several types of AMCs have been proposed [10-13]. These structures can be realized using Printed Circuit Board (PCB) techniques. In 2001, Yang et al. [14] introduced the Uniplanar Compact Photonic Band Gap (UC PBG) structure which has the advantage of ease of fabrication (since it does not involve vias) by etching a periodic pattern on a substrate backed by a ground plane.

On the other hand, wearable and conformal wireless systems are becoming very popular nowadays. Furthermore, the integration of AMC structures and metamaterial with such systems is a growing field of research $[15,16]$; it is expected to be commercially available soon. Textile materials, flexible substrates and planar structures have been recently proposed to integrate antennas into fabrics or curvy planes. In [17-19], wearable antennas for WLAN, telemedicine, GPS, and Bluetooth applications were proposed. Noticeably, maintaining a flat profile for antenna elements based on flexible materials is not feasible. Hence, it is important to analyze the performance of the antennas and any type of integrated structures under bending conditions. Previous studies were mainly focused on the effect of bending on the performance of wearable antennas based on simulation and experimental results $[20,21]$. To the best of the authors' knowledge, no research has been reported on the performance of UCPBG or AMC structures under bending conditions.

In this paper, we specifically consider the performance of the conventional UC-PBG structure proposed in [13] operating as an AMC 
under different extents of bending. The effect of bending on the band gap characteristics is out of the scope of this paper and will be the subject of a future research.

Geometrical modelling of the UC-PBG structure along with the necessary numerical setup is introduced in Section 2, where the geometry of the lattice is explained. The simulated performance of the AMC structure under different extents of bending is discussed in Section 3. Finally, the paper is concluded in Section 4.

\section{MODELLING AND SIMULATION SETUP}

\subsection{A-modelling}

Modelling of UC-PBG geometry along with numerical analysis have been carried out using the commercial software package CST Microwave Studio which is based on the Finite Integration Technique (FIT) [22]. The conventional UC-PBG structure is realized by a two-dimensional metallic pattern with each element comprising a central square metal patch with four smaller pads on the corners and four narrow connected branches. The metallic pattern is etched on a substrate of a specific thickness and backed by a ground plane. The combination of the metallic pattern with the ground plane forms a distributed $L C$ network. The narrow branches, with the connection insets, introduce an inductance while the gaps between adjacent pads give rise to a capacitance. The resonance frequency is determined by the series inductive-capacitive elements in addition to shunt capacitances realized by the metallic pattern and the ground plane [13]. It is worth mentioning that the performance of AMCs is mainly characterized by their resonant frequency and bandwidth which are functions of the substrate thickness, dielectric constant and structure geometry.

The dimensions of the UC-PBG are shown in Figure 1. The substrate used in the model is assumed to be flexible, has a dielectric constant of 1 , it is also assumed to be lossless (modelled as vacuum for simplicity) with a thickness of $0.9 \mathrm{~mm}$. To decrease the simulation time, the structure's ground plane and metallic pattern were modelled as PECs.

\subsection{B-simulation Setup}

The typical UC-PBG unit cell model and simulation setup of the structure are depicted in Figure 1. To excite the structure, a source of an incident plane wave impinges from the top of the vacuum box is defined. By setting the right boundary conditions (Perfect 


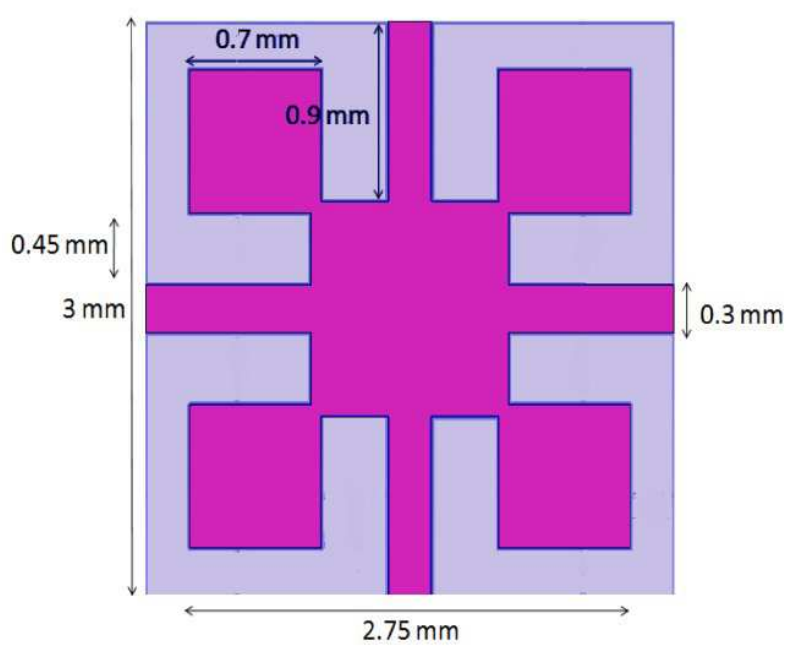

Figure 1. Dimensions of the UC-PBG unit cell.

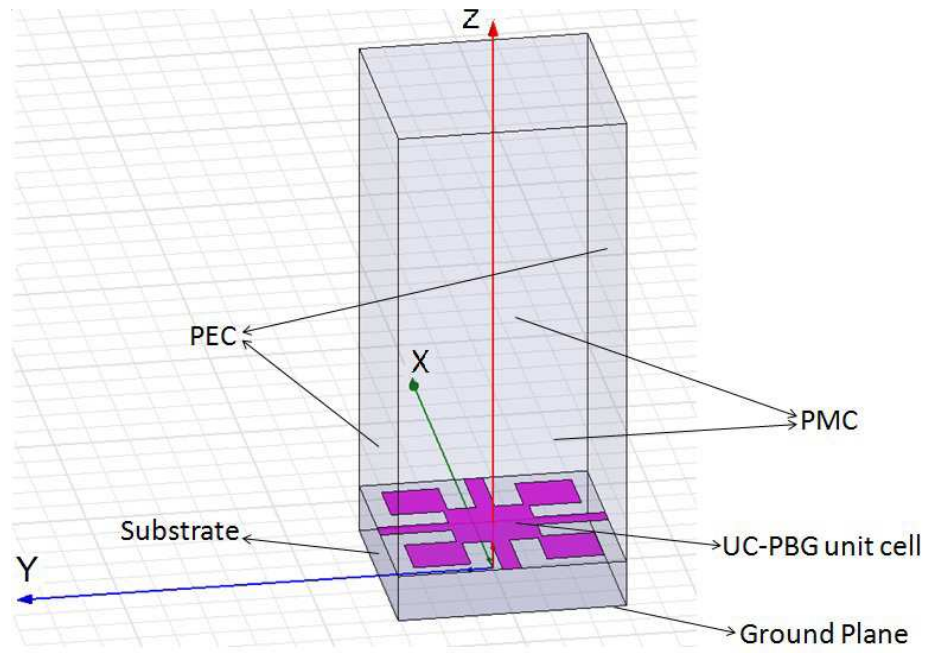

Figure 2. Numerical setup of a UC-PBG unit cell.

Electric Conductor (PEC) and Perfect Magnetic Conductor (PMC) boundaries imposed as sidewalls), Transverse Electromagnetic Mode (TEM) incident wave can be enforced. The height of the vacuum box is normally chosen to be bigger than $\lambda / 4$. It is worth mentioning here that the box height should be de-embedded by the same amount to yield the right TEM incident's phase reference. When simulating normal 
(flat) AMC structures the unit cell is terminated at the bottom by a PEC boundary to represent the ground plane. It should be noted that this boundary is not valid when simulating a curvy structure. Instead, a model object should be considered and the bottom boundary should be set to "open". For AMC analysis, the reflection phase response is sufficient to determine the basic performance characteristics such as resonant frequency and bandwidth.

In this study, we chose a $4 \times 4$ lattice of UCPBG units as an example since it is irrational to perform the bending effect on a single unit cell as the effect will be duplicated in the $x$ and $y$ direction (in a wavy fashion). To be more practical, the effect o bending should be applied on the same number of cells for the application under study.

The reflection phase of an AMC structure varies continuously from $-180^{\circ}$ to $+180^{\circ}$ as frequency sweeps. AMC resonance frequency is achieved when the phase crosses the zero point. Generally, the operational bandwidth of an AMC is generally defined as $-90^{\circ}$ to $+90^{\circ}$ on both sides of the resonance frequency. It can be seen from the reflection phase of the unit cell (flat case) that the AMC operational frequency is $38 \mathrm{GHz}$ with a bandwidth of $6.7 \mathrm{GHz}(17.6 \%)$. To validate our analysis, the $4 \times 4$ lattice (shown in Figure 3 ) was also simulated under the same numerical setup, identical reflection phase was observed.

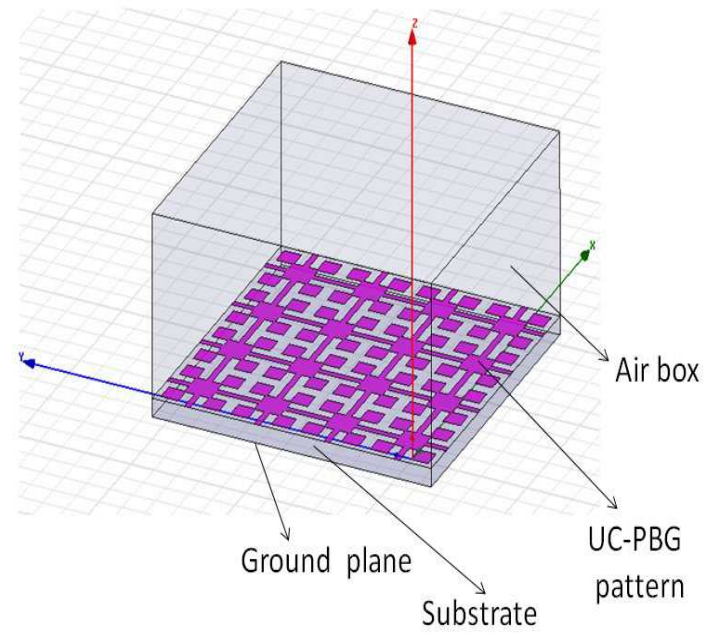

Figure 3. A $4 \times 4$ lattice of UC-PBG. 


\section{CONFORMAL AMC PERFORMANCE}

In order to bend the UC-PBG structure, a CST Microwave Studio "bend sheet" tool was used along with "thicken sheet". A vacuum cylinder with a parametric radius to control the bending extent was modelled in order to roll the combination of metallic pattern, substrate and ground plane. Obviously, a larger radius introduces less curvature and vice versa.

In this paper, two bending degrees are considered. Slight bending when the structure is bent on a $7 \mathrm{~mm}$ radius and $4 \mathrm{~mm}$ radius for an extreme bending degree. Higher or lower degrees of bending can be achieved by controlling the vacuum cylinder's radius. It should be noted that normal incidence and reflection are assumed for the specified AMC structure since it is mainly utilized for antenna applications which are positioned in a very close proximity (within $0.1 \lambda$ ).

Here, we would like to pinpoint important remarks that should be followed when simulating bended AMC structures:

The bended UC-PBG should be considered as a non-periodic structure since periodic boundary conditions will lead to a non realistic model.

The boundary conditions for a non periodic model are PEC for two opposite walls and "open" boundary conditions are set to the other opposite walls where the bended edges of the structure are located (as shown in Figure 4). It should be noted that these boundary conditions impose non periodicity in the $x$-axis only

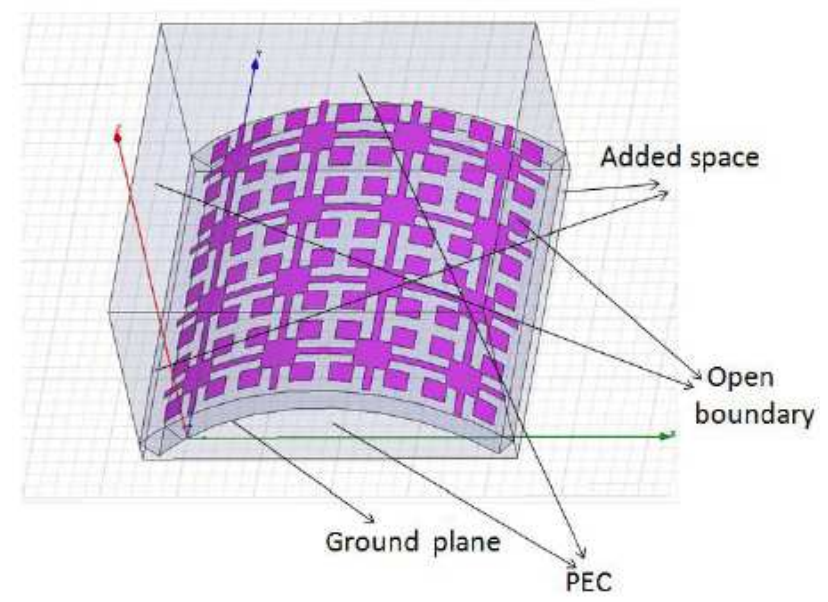

Figure 4. Bent UC-PBG on a cylinder of $7 \mathrm{~mm}$ radius. 
while the periodicity still holds in the $y$-axis. Obviously, the boundaries could be interchanged accordingly depending on the structure symmetry.

Added space should be inserted to the open boundaries sides to minimize the higher order modes caused by the boundaries sudden transition.

The amount of de-embedding should be decreased accordingly when the degree of bending is higher as the curvature yields to a higher structure tip.

The width of the vacuum box along with the added space should be adjusted when the structure is modified; intuitively, the structure's effective width decreases as the curvature increases.

As an example, a slightly bended structure (on a $7 \mathrm{~mm}$ radius) is shown in Figure 4.

For comparison purposes, the reflection phases of the three aforementioned scenarios (starting with the flat case and ends with the highest bending degree) are shown below in Figures 5, 6, and 7, respectively. Operational frequencies and bandwidths are extracted from the reflection phase profiles and are depicted in Table 1.

As can be inferred from the reflection phase profiles, the AMC resonant frequency increases and the bandwidth decreases when the angle of incidence is increased. This phenomenon occurs since the

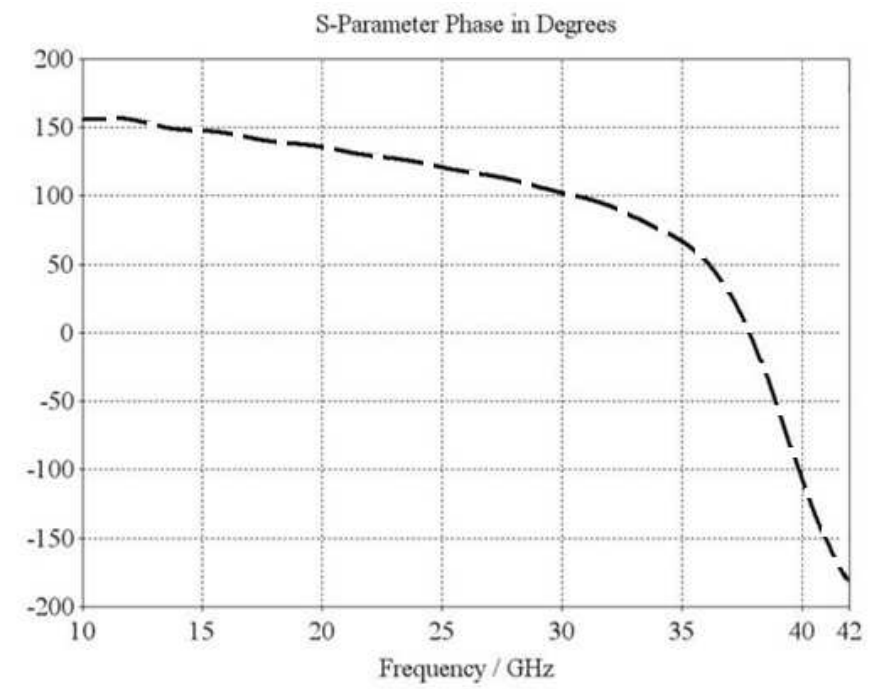

Figure 5. Computed reflection phase for the UC-PBG under study (flat case). 
Table 1. UC-PBG performance under bending effect.

\begin{tabular}{|c|c|c|}
\hline Degree of bending & AMC frequency $\mathbf{( G H z )}$ & Bandwidth \% \\
\hline Flat & 38 & 17.6 \\
\hline Bent on $r=7 \mathrm{~mm}$ & 39.1 & 13.5 \\
\hline Bent on $r=4 \mathrm{~mm}$ & 40.7 & 2.7 \\
\hline
\end{tabular}

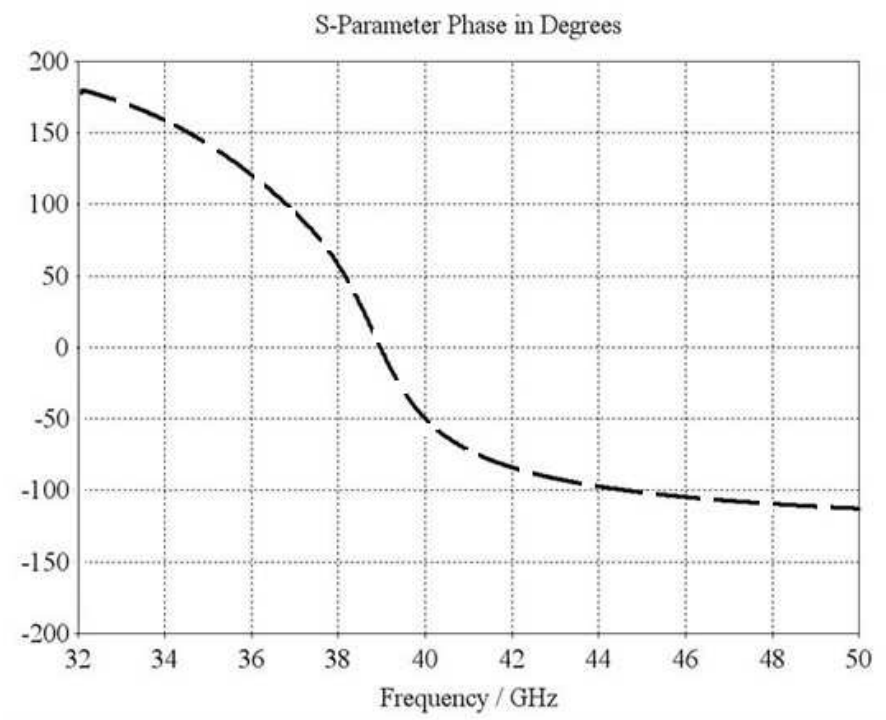

Figure 6. Computed reflection phase for the UC-PBG bent on a cylinder of a $7 \mathrm{~mm}$ radius.

incident wave becomes "oblique" with reference to the bended surface. As can be seen in Figure 6, for a smaller degree of curvature (i.e., angle of incidence), the AMC resonant frequency is slightly shifted to the right side $(1.1 \mathrm{GHz})$, while the bandwidth is slightly reduced. For a higher degree of curvature (i.e., higher angle of incidence), more shift to a higher $\mathrm{AMC}$ resonant frequency is observed $(2.7 \mathrm{GHz})$, while the reflection phase becomes steeper which yields to a further reduction in the operational bandwidth (as shown in Figure 7). The shift in resonant frequency and reduction in bandwidth are due to the fact that the equivalent free space impedance $Z_{o}$ is decreased as the incident angle is increased since they are proportional to the surface impedance of the AMC [23]. Hence, the effective inductance and capacitance will be affected accordingly. As is well known, the bandwidth is directly proportional to the inductance and the resonant frequency is inversely proportional to $1 / L C$. 


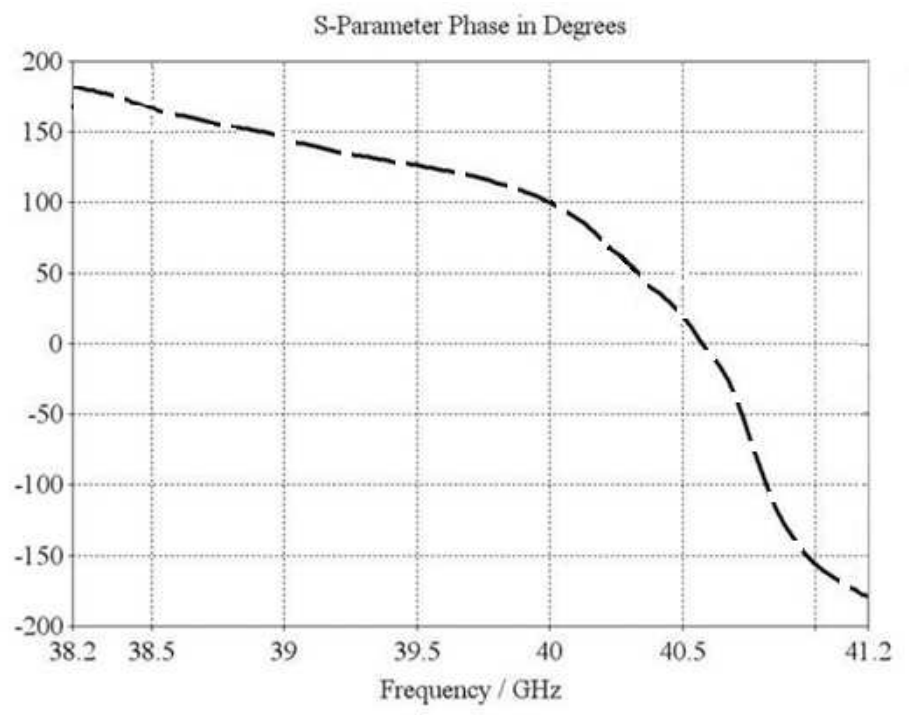

Figure 7. Computed reflection phase for the UC-PBG bent on a cylinder of a $4 \mathrm{~mm}$ radius.

\section{CONCLUSION}

The performance of the conventional UC-PBG structure operating as an AMC was investigated under different bending extents. Our numerical analysis shows that the reflection phase profile which is employed to infer the AMC resonant frequency and bandwidth is affected under different bending degrees. A comparison between the flat case and two bending degrees was conducted parametrically to show how the performance is affected. A shift to a higher AMC resonance frequency is observed in addition to degradation in the operational bandwidth when the degree of bending is increased. It is concluded that this type of study is important when designing AMC structures for conformal applications where the operating frequency and bandwidth are the cornerstones of the performance.

\section{ACKNOWLEDGMENT}

This research was funded in part by the National Science Foundation Grants (NSF EPSCoR) EPS-0701890 and CNS-0619069. 


\section{REFERENCES}

1. Sievenpiper, D. F., M. E. Sickmiller, and E. Yablonovitch, "3D wire mesh photonic crystals," Phys. Rev. Lett. B, Condens. Matter, Vol. 76, 2480-2483, Apr. 1996.

2. Shumpert, J., T. Ellis, G. Rebeiz, and L. Katehi, "Microwave and millimeter-wave propagation in photonic bandgap structures," IEEE AP-S/URSI Symp. Dig., 678, 1997.

3. Qian, Y., V. Radisic, and T. Itoh, "Simulation and experiment of photonic bandgap structures for microstrip circuits," IEEE APMC. Symp. Dig., 585-588, Hong Kong, Dec. 2-5, 1997.

4. Brown, E. R., C. D. Parker, and E. Yablonovitch, "Radiation properties of a planar antenna on a photonic-crystal substrate," J. Opt. Soc. Amer. B, Opt. Phys., Vol. 10, 404-407, Feb. 1993.

5. Sigalas, M. M., R. Biswas, and K. M. Ho, "Theoretical study of dipole antennas on photonic band-gap materials," Microwave Opt. Technol. Lett., Vol. 13, 205-209, Nov. 1996.

6. Yang, F. and Y. Rahmat-Samii, Electromagnetic Band-gap Structures in Antenna Engineering, Cambridge Univ. Press, Cambridge, UK, 2008.

7. Goussetis, G., A. P. Feresidis, and J. C. Vardaxoglou, "Tailoring the AMC and EBG characteristics of periodic metallic arrays printed on grounded dielectric substrate," IEEE Transactions on Antennas and Propagation, Vol. 54, No. 1, 82-89, Jan. 2006.

8. Zhu, S. and R. J. Langley, "Dual band wearable antennas over EBG substrate," Electron. Lett., Vol. 43, No. 3, 141-143, Feb. 2007.

9. Sievenpiper, D., L. J. Zhang, R. F. J. Broas, N. G. Alexopolous, and E. Yablonovitch, "High impedance electromagnetic surfaces with a forbidden frequency band," IEEE Trans. Microw. Theory Tech., Vol. 47, No. 11, 2059-2074, Nov. 1999.

10. Yang, L., M. Y. Fan, F. L. Chen, J. Z. She, and Z. H. Feng, "A novel compact electromagnetic bandgap (EBG) structure and its applications for microwave circuits," IEEE Trans. Microw. Theory Tech., Vol. 53, No. 1, 183-190, Jan. 2005.

11. Hosseinipanah, M. and Q. Wu, "Miniaturised high-impedance surface with high angular stability of resonant frequency," Electron. Lett., Vol. 45, No. 24, 1204-1206, Nov. 2009.

12. Kim, Y., F. Yang, and A. Z. Elsherbeni, "Compact artificial magnetic conductor designs using planar square spiral geometries," Progress In Electromagnetics Research, Vol. 77, 43-54, 2007. 
13. Yousefi, L., H. Attia, and O. M. Ramahi, "Broadband experimental characterization of artificial magnetic materials based on a microstrip line method," Progress In Electromagnetics Research, Vol. 90, 1-13, 2009.

14. Yang, F. R., K. P. Ma, Y. X. Qian, and T. Itoh, "A uniplanar compact photonic-bandgap (UC-PBG) structure and its applications for microwave circuit," IEEE Trans. Microw. Theory Tech., Vol. 47, No. 8, 1509-1514, Aug. 1999.

15. Salonen, P. O., F. Yang, Y. Rahmat-Samii, and M. Kivikoski, "WEBGA - Wearable electromagnetic band-gap antenna," Proc. IEEE Antennas Propag. Soc. Int. Symp., Vol. 1, 455-459, 2004.

16. Salonen, P., Y. Rahmat-Samii, M. Schaffrath, and M. Kivikoski, "Effect of textile materials on wearable antenna performance: A case study of GPS antennas," Proc. IEEE Antennas Propag. Soc. Int. Symp., Vol. 1, 459-462, 2004.

17. Massey, P. J., "Mobile phone antennas integrated within clothing," Proc. IEE 11th Int. Conf. Antennas Propag. (ICAP'01), Vol. 1, 344-347, Manchester, UK, 2001.

18. Salonen, P., M. Keskialammi, and L. Sydanheimo, "A low cost $2.45 \mathrm{GHz}$ photonic band gap patch antenna for wearable systems," Proc. IEE 11th Int. Conf. Antennas Propag. (ICAP'01), Vol. 2, 719-723, Manchester, UK, 2001.

19. Khaleel, H. R., H. M. Al-Rizzo, D. G. Rucker, and T. A. Elwi, "Wearable yagi microstrip antenna for telemedicine applications," IEEE RWS Symp., 280-283, New Orleans, USA, 2010.

20. Locher, I., M. Klemm, T. Kirstein, and G. Troster, "Design and characterization of purely textile patch antennas," IEEE Transactions on Advanced Packaging, Vol. 29, No. 4, 777-788, Nov. 2006.

21. Salonen, P. and Y. Rahmat-Samii, "Textile antennas: Effects of antenna bending on input matching and impedance bandwidth," IEEE Aerospace and Electronic Systems Magazine, Vol. 22, No. 12, 18-22, Dec. 2007.

22. CST Microwave Studio, http://www.cst.com.

23. Luukkonen, O., C. Simovski, G. Granet, G. Goussetis, D. Lioubtchenko, A. V. Raisanen, and S. A. Tretyakov, "Simple and accurate analytical model of planar grids and high-impedance surfaces comprising metal strips or patches," IEEE Transactions on Antennas and Propagation, Vol. 56, No. 6, 1624-1632, Jun. 2008. 